\title{
Positive Maternal Attitudes during Infancy and a Positive Lifestyle May Be Associated with Beneficial Epigenetic Alterations and Prevent or Improve the Symptoms of Alzheimer's Disease
}

\author{
Riichiro Ishida \\ RIKEN (The Ishida-Riichiro Institute), Niigata, Japan \\ Email: ishida-riichiro@yf6.so-net.ne.jp
}

How to cite this paper: Ishida, R. (2019). Positive Maternal Attitudes during Infancy and a Positive Lifestyle May Be Associated with Beneficial Epigenetic Alterations and Prevent or Improve the Symptoms of Alzheimer's Disease. Psychology, 10, 953-957. https://doi.org/10.4236/psych.2019.107062

Received: May 28, 2019

Accepted: June 17, 2019

Published: June 20, 2019

Copyright $\odot 2019$ by author(s) and Scientific Research Publishing Inc. This work is licensed under the Creative Commons Attribution International License (CC BY 4.0).

http://creativecommons.org/licenses/by/4.0/

\section{(c) (i) Open Access}

\begin{abstract}
Alzheimer's disease (AD) is the most common form of dementia and the cause of premature senility, resulting in progressive mental deterioration and neurobehavioral deficits. $\mathrm{AD}$ is caused by the accumulation of $\beta$-amyloid protein, which leads to the accumulation of phosphorylated protein causing neuronal death in various areas of the brain, such as the hippocampus, which is associated with memory. This process is associated with oxidative stress caused by excessive reactive oxygen species. Serotonin may contribute to a greater ability to manage stressful events via growth factors. Melatonin may enhance the regeneration of tissues with the help of various growth factors. This process is associated with epigenetic alterations. Warm-hearted maternal attitudes during infancy and a positive lifestyle, which includes a well-balanced diet, moderate aerobic exercise, purpose in life ("ikigai"), and other warm-hearted human relations, may increase the secretion of serotonin and melatonin along with the well-balanced secretion of neurotransmitters and hormones. Increased secretion of serotonin and melatonin may contribute to a better ability to manage stressful events, and may enhance the prevention and treatment of the symptoms of $\mathrm{AD}$ by decreasing levels of $\beta$-amyloid and facilitating the regeneration of tissue in the hippocampus.
\end{abstract}

\section{Keywords}

$\beta$-amyloid, Alzheimer's Disease, Stress Management, Stressful Events, Regeneration of Tissue in the Hippocampus, Epigenetics, Maternal Attitudes, Lifestyle 


\section{Introduction}

Alzheimer's disease (AD) is the most common form of dementia and the cause of premature senility, resulting in progressive mental deterioration and neurobehavioral deficits (Shukla, Govitrapong, Boontem, Reiter, \& Satayavivad, 2017). AD is caused by the accumulation of $\beta$-amyloid protein leading to accumulation of phosphorylated protein causing the death of neurons in each area of the brain, such as the hippocampus, which is associated with memory (Yasuda, 2013). Reactive oxygen species, which are produced by activities of the brain and body, cause oxidant stress, which, in turn, results in the production of $\beta$-amyloid (Yasuda, 2013). A genetic factor, i.e., apolipoprotein $\mathrm{E}$, also influences deposition of $\beta$-amyloid (Yasuda, 2013). $\beta$-amyloid is decomposed by enzyme neprilysin (NEP), i.e., neutral endopeptidase (Yasuda, 2013; Tahira, 2014). Exercise is one of the factors that contribute to a greater ability to manage stressful events and is known to increase the production of neprilysin (Yasuda, 2013). This evidence suggests that a greater ability to manage stressful events helps to inhibit the production of excessive reactive oxygen species that cause oxidant stress, and then increase the production of sufficient NEP or neutral endopeptidase, which inhibits the accumulation of $\beta$-amyloid leading to the prevention and improvement of the symptoms of $\mathrm{AD}$. The autonomic nervous system and endocrine system respond to stressful events via neurotransmitters and hormones (Ishida, 2019). Recently, the generation of neurons by stem cells has been observed in the lateral ventricle and hippocampus of adults (Nakajima, 2013). This indicates that it may be possible to generate neurons at any age. Epigenetics is the study of changes to gene expression in response to chemicals, e.g., deoxyribonucleic acid (DNA) demethylation and histone acetylation. These changes do not modify the genomic sequence, but are inheritable and reversible (Ishida, 2019). Serotonin (5-hydroxytryptamine; $\mathrm{C}_{10} \mathrm{H}_{12} \mathrm{~N}_{2} \mathrm{O}$ ), a neurotransmitter, and melatonin (N-acetyl-5-methoxytryptamine; $\mathrm{C}_{13} \mathrm{H}_{16} \mathrm{~N}_{2} \mathrm{O}_{2}$ ), a hormone, have been shown to contribute to positive epigenetic alterations (Jenwitheesuk, Nopparat, Mukda, Wongchitrat, \& Govitrapong, 2014; Ishida, 2019). This suggests that it may be possible to regenerate neurons in a damaged hippocampus via epigenetic mechanisms. Therefore, it is possible that $\mathrm{AD}$ can be prevented and improved by improving the body's ability to manage stressful events. Recently, positive maternal attitudes during infancy and leading a positive lifestyle with a focus on improving the management of stressful events have been studied (Ota, 2013; Ishida, 2019). A positive lifestyle includes purpose in life (PIL), which is also referred to as "ikigai", moderate aerobic exercise, and a well-balanced diet (Ishida, 2019). The concept of PIL comes from existentialism in Europe, and the term ikigai has a long history of use in daily life in Japan (Ishida, 2019). Both terms include the common sense notions that everything changes, and everyone only has one life that should have purpose or meaning to them (Ishida, 2019). The desire to establish a meaning in life or to live a life worth living is natural and represents the intrinsic function of the prefrontal 
cortex (PFC) (Ishida, 2019). PIL/ikigai develops through positive experiences and accompanying impressions including warm-hearted human relationships, holding people in respect, succeeding in a challenging goal that a person sets by him/herself, and spending sufficient time in beautiful natural surroundings (Ishida, 2019). Therefore, the author proposes ways of preventing and improving the symptoms of AD based on PFC function, epigenetic alteration, serotonin, and melatonin. This process may increase the quality of life (QOL) of not only patients with $\mathrm{AD}$, but also healthy people.

\section{PFC Function}

The PFC connects various areas of the brain via a neuronal network. The functions of the PFC include the integration of information from other areas of the brain, planning, decision-making, inference and imaging, and the establishment of human relationships (Nolen-Hoeksema, Fredrickson, Loftus, \& Luts, 2014; Ishida, 2019). PFC function contributes to the ability to manage stressful events, thus contributing to human prosperity. In addition, a person's level of maturity (i.e., whether an individual's personality demonstrates "human nature" or "humanity" contributes not only to their own happiness, but also to others' happiness), is dependent on his/her PFC function and is directly related to the function of neurotransmitters and hormones.

\section{Epigenetic Alteration}

Generally speaking, DNA methylation contributes to gene repression, while DNA demethylation contributes to gene expression (Ota, 2013; Obana-Koshino, Ono, Miura, Sakai, Uchida, Nakamura et al., 2015). Histone acetylation contributes to the transcription and translation of DNA (Ota, 2013; Obana-Koshino et al., 2015). Therefore, understanding the influence of serotonin and melatonin on epigenetic may be an important contribution to the prevention and improvement of the symptoms of $\mathrm{AD}$.

\section{Serotonin}

Serotonin is a neurotransmitter that is involved in homeostatic regulation and it facilitates prefrontal inhibition. Specifically, serotonin modulates emotions by regulating the secretion of other neurotransmitters (Ota, 2013; Kabasawa, 2017). Serotonin is synthesized from tryptophan, which is an essential amino acid that is obtained from food, and is secreted by various organs (Ota, 2013). Serotonin can improve resistance to stress through a process that depends on the following chemical response (Ota, 2013). Increased levels of serotonin induce the expression of nerve growth factor (NGF)-derived protein A, which decreases the level of DNA methylation on the promoter of the glucocorticoid (GR) receptor gene and promotes histone acetylation. Consequently, GR receptor expression increases. GR is a steroid hormone that is secreted by the adrenal cortex. It is involved in the breakdown of sugar, and contributes to emotional stability. GR al- 
so acts as a stress hormone by conferring greater resistance to stress. Decreased serotonin levels result in lower GR receptor levels, and contribute to decreased stress tolerance. Cognition and emotion, which are associated with neurotransmitters and hormones, are indicators of overall welfare, i.e., positive emotions and cognition are associated with successes, while negative emotions and cognition are associated with failures in adapting to changing environments. In summary, well-balanced synthesis and secretion of neurotransmitters, especially sufficient serotonin, may inhibit excessive reactive oxygen species that are accompanied by excessive oxidant stress, leading to the inhibition of the accumulation of $\beta$-amyloid, resulting in the prevention and improvement of the symptoms of AD.

\section{Melatonin}

Melatonin is a hormone that is involved in regulating the circadian rhythm, free radical scavenging, anti-oxidation, the immune system, the inhibition of tumors, the generation of new vascular networks and cells, DNA repair, the regulation of body temperature, and bone induction activity (Obana-Koshino et al., 2015; Kabasawa, 2017). Melatonin is syntesized from serotonin. Epidermal growth factor (EGF) is involved in regulating cell growth and proliferation, while NGF is involved in the extension of the axis cylinder of nerves, the induction of neurotransmitter synthesis, and the maintenance of nerve cell functions, i.e., the restoration of cells following cell damage (Obana-Koshino et al., 2015). Certain vitamins and minerals also act as enzymes that enhance chemical responses (Nolen-Hoeksema et al., 2014). Melatonin causes epigenetic effects against cancer cells by modulating both DNA methylation and histone acetylation pathways (Jenwitheesuk et al., 2014). When neural stem cells were treated with melatonin, significantly increased histone acetylation and enhanced histone deacetylase isoforms were observed in response to melatonin-induced histone hyperacetylation (Jenwitheesuk et al., 2014). In summary, melatonin, EGF, and NGF may contribute to the regeneration of tissue in the hippocampus.

\section{Limitations and Future Studies}

The limitations of factors influencing epigenetic alterations have not yet been clearly defined. Therefore, future studies are required to obtain more data and empirical evidence on the relationship between experiences and epigenetic alterations, the roles of neurotransmitters, hormones, vitamins, minerals, and light catalysts, such as sunlight, and the effects of hydroxymethylcytosine on the process of DNA demethylation and histone acetylation.

\section{Conclusion}

Serotonin may contribute to a greater ability to manage stressful events via growth factors. Melatonin may enhance the regeneration of tissues with the help of various growth factors. Warm-hearted maternal attitudes during infancy and 
a positive lifestyle, which includes a well-balanced diet, moderate aerobic exercise, PIL/ikigai, and other warm-hearted human relationships, contribute to the greater ability to manage stressful events leading to steady emotions and cognitions, and may increase the secretion of serotonin and melatonin. Increased secretion of serotonin and melatonin may contribute to a greater ability to manage stressful events, and may enhance the prevention and treatment of the symptoms of $\mathrm{AD}$ by decreasing $\beta$-amyloid levels and facilitating the regeneration of tissue in the hippocampus. All persons should know that positive maternal attitudes during infancy and a positive lifestyle may influence positive epigenetic alteration leading to preventing and improving the symptoms of $\mathrm{AD}$.

\section{Conflicts of Interest}

The author declares no conflicts of interest regarding the publication of this paper.

\section{References}

Ishida, R. (2019). Positive Maternal Attitudes during Infancy and a Positive Lifestyle May Be Associated with Beneficial Epigenetic Alterations and Improved Oral Health. Psychology, 10, 742-747. https://doi.org/10.4236/psych.2019.105048

Jenwitheesuk, A., Nopparat, C., Mukda, S., Wongchitrat, P., \& Govitrapong, P. (2014). Melatonin Regulates Aging and Neurodegeneration through Energy Metabolism, Epigenetics, Autophagy, and Circadian Rhythm Pathways. International Journal of Molecular Sciences, 15, 16848-16884. https://doi.org/10.3390/ijms150916848

Kabasawa (2017). Noh wo Saitekikasureba Nohryoku wa 2 bai ni naru. Tokyo: Bunkyosha.

Nakajima, K. (2013). Shinkei Hassei. In S. Tajima (Ed.)., Epigenetics. Kyoto: Kagakudohjin.

Nolen-Hoeksema, S. N., Fredrickson, B. L., Loftus, G. R., \& Luts, C. (2014). Atkinson \& Hilgard's Introduction to Psychology (16th ed.). Stamford, CT: Cengage Learning EMEA.

Obana-Koshino, A., Ono, H., Miura, J., Sakai, M., Uchida, H., Nakamura, W. et al. (2015). Melatonin Inhibits Embryonic Salivary Gland Branching Morphogenesis by Regulating Both Epithelial Cell Adhesion and Morphology. PLoS ONE, 10, e0119960. https://doi.org/10.1371/journal.pone.0119960

Ota, K. (2013). Epigenomu to Seimei. Tokyo: Kodansha.

Shukla, M., Govitrapong, P., Boontem, P., Reiter, R. J., \& Satayavivad, J. (2017). Mechanism of Melatonin in Alleviating Alzheimer's Disease. Current Neuropharmacology, 15, 1010-1031. https://doi.org/10.2174/1570159X15666170313123454

Tahira, T. (2014). Noh (Ninchishoh). In N. Ishii, \& N. Maruyama (Eds.), Biomedical Gerontology (Rohka no Seibutsugaku). Kyoto: Kagakudohjin.

Yasuda, K. (2013). Ninnchishyoh Naotta. Tokyo: Shufunotomoshya. 